3. R. P. Agarwal, "Some hypergeometric identities," Ann. Soc. Sci. Bruxelles Sér. I, v. 68,1953 , pp. 186-202. MR 15, 421 .

4. W. HAнN, "Úber die höheren Heineschen Reihen und eine einheitliche Theorie der sogenannten speziellen Functionen," Math. Nachr., v. 3, 1950, pp. 257-294. MR 12, 711.

5. W. HAHN, "Beiträge zur Theorie der Heineschen Reihen," Math. Nachr., v. 2, 1949, pp. 340-379. MR 11, 720 .

6. F. H. JACKSON, “q-difference equations," Amer. J. Math., v. 32, 1910, pp. 305-314.

7. J. L. FinLd \& J. WIMP, "Expansion of hypergeometric functions in hypergeometric functions," Math. Comp., v. 15, 1961, pp. 390-395.

8. L. Toscano, "Teorema di moltiplicazione sulle funzioni ipergeometriche generalizzate," Ann. Mat. Pura Appl., (4), v. 44, 1956, pp. 269-288. MR 17, 1206.

9. Y. L. LUKE \& R. L. Coleman, "Expansion of hypergeometric functions in series of other hypergeometric functions," Math. Comp., v. 15, 1961, pp. 233-238.

10. F. J. W. WHIPpLe, "Some transformations of generalised hypergeometric series," Proc. London Math. Soc., v. 22, 1927, pp. 257-272.

\title{
Boundedness of Difference Kernels of Bessel and Fourier Series
}

\section{By Shih-Hsiung Tung}

1. Introduction. Let $\alpha_{m}, m=1,2, \cdots$, be positive zeros of Bessel functions $J_{\nu}(x)$ of the first kind of order $\nu \geqq-\frac{1}{2}$, arranged in increasing order. The kernels of Bessel and Fourier cosine series on $[0,1]$ are denoted as

$$
B_{M}(x ; t)=\sum_{m=1}^{M} 2 t J_{\nu}\left(\alpha_{m} x\right) J_{\nu}\left(\alpha_{m} t\right) J_{\nu+1}^{2}\left(\alpha_{m}\right)
$$

and

$$
C_{M} \equiv C_{M}(x ; t)=1+\sum_{m=1}^{M} 2 \cos (m \pi x) \cos (m \pi t)
$$

We define the difference kernel to be

$$
D_{M}(x ; t)=B_{M}(x ; t)-C_{M}(x ; t) .
$$

Two series $S=\sum s_{i}$ and $T=\sum t_{i}$ are said to be equiconvergent if $\lim _{n \rightarrow \infty}\left(S_{n}-T_{n}\right)=0$, where $S_{n}$ and $T_{n}$ are partial sums of the first $n$ terms of the series.

Here we study the boundedness (Theorems 1 and 2) of the difference kernel and the equiconvergence (Theorem 3) of Bessel series of a Lebesgue integrable function on $[0,1]$ and its corresponding Fourier cosine series. The proof of the boundedness of the difference kernel of two series is mainly based on the application of the asymptotic expansion of Bessel functions and their zeros. The equiconvergence theorem, which is a direct application of Theorem 2, is a stronger result obtained by a simple and straightforward proof comparable to the analogous ones given in [6] and [8]. We notice that the cosine series may equally well be replaced by a sine or sine and cosine series.

2. Preliminaries. The following results are needed later.

LeмmA 1. If $a$ is real, $b \geqq 0$ and $0<\eta \leqq y-k \leqq 1-\eta<1$ for some integer

Received May 20, 1965 
$k$, then $\sum_{m=1}^{M} m^{-b} \sin (2 m \pi y+a)$ and $\sum_{m=1}^{M} m^{-b} \cos (2 m \pi y+a)$ are bounded independently of $a, y$, and $M$.

Proof. Let $y=k+\zeta$ and $0<\eta \leqq \zeta \leqq 1-\eta<1$. For the case $b=0,\left|\sum_{m=1}^{M} \sin (2 m \pi y+a)\right| \leqq(\sin \pi \zeta)^{-1}$ is obtained from the formula

$$
2 \sin (\pi \zeta) \sum_{m=1}^{M} \sin (2 m \pi \zeta+a)=2 \sin (M \pi \zeta) \sin [(M+1) \pi \zeta+a] .
$$

Similarly, $\left|\sum_{m=1}^{M} \cos (2 m \pi y+a)\right| \leqq(\sin \pi \zeta)^{-1}$. The case $b>0$ follows from the case $b=0$ by, applying Dirichlet's test [3, p. 347].

The following result was given in $[1$, p. 382].

LemMa 2. $\sum_{m=1}^{M} m^{-1} \sin (m+a) \zeta$ is bounded independently of $a$, and $M$, where $0 \leqq \zeta \leqq 2 \pi-\eta, \eta>0$ and $a \geqq-\frac{1}{2}$.

It is known that [7, p. 199], for large value of $x>0$,

$$
J_{\nu}(x)=\sqrt{ }\left(\frac{2}{\pi x}\right)\left[\cos p(x)+\frac{\nu^{2}-\frac{1}{4}}{2 x} \sin p(x)+O\left(x^{-2}\right)\right],
$$

where

$$
p(x)=-x+\frac{1}{4}(2 \nu+1) \pi .
$$

The $m$ th zero $\alpha_{m}$ of $J_{\nu}(x)$ is given by asymptotic expansion [7, p. 506]

$$
\alpha_{m}=\left(m+\nu / 2-\frac{1}{4}\right) \pi+\beta_{m},
$$

where

$$
\beta_{m}=\frac{1-4 \nu^{2}}{8\left(m+\nu / 2-\frac{1}{4}\right)}+O\left(m^{-3}\right)=O\left(m^{-1}\right) .
$$

From (5), (6) and (7), for $x=\alpha_{m}, \cos p\left(\alpha_{m}\right)=(-1)^{m-1} \cos \beta_{m}$ and $\sin p\left(\alpha_{m}\right)=$ $(-1)^{m} \sin \beta_{m}$. By substituting these in (4) and using (6) and (7), we obtain by a calculation

$$
J_{p+1}^{2}\left(\alpha_{m}\right)=\frac{1}{2} \pi \alpha_{m}\left[1+O\left(m^{-2}\right)\right] .
$$

From Eqs. (4), (6) and (8), for $x, t>0$ and large $m$, we have

$$
\begin{aligned}
b_{m}(x ; t)=2 t J_{\nu}\left(\alpha_{m} x\right) J_{\nu}\left(\alpha_{m} t\right) J_{\nu+1}^{2}\left(\alpha_{m}\right) & \\
=(t / x)^{1 / 2}\left[1+O\left(m^{-2}\right)\right]\left\{2 \cos p\left(\alpha_{m} x\right) \cos p\left(\alpha_{m} t\right)\right. & \\
& +\frac{\nu^{2}-\frac{1}{4}}{2 \alpha_{m}}\left[\frac{2}{x} \sin p\left(\alpha_{m} x\right) \cos p\left(\alpha_{m} t\right)\right. \\
& \left.\left.+\frac{2}{t} \cos p\left(\alpha_{m} x\right) \sin p\left(\alpha_{m} t\right)\right]+O\left(m^{-2}\right)\right\} .
\end{aligned}
$$

For fixed $x \in(0,1)$, we take $\delta$ such that $0<\delta \leqq \min \{x / 2,(1-x) / 2\}$. And from $(t / x)^{1 / 2}=(1+(t-x) / x)^{1 / 2}$, we have

$$
(t / x)^{1 / 2}=1+\frac{1}{2}\left(1+\theta \frac{t-x}{x}\right)^{-1 / 2} \frac{t-x}{x}
$$


where $0<\theta<1$. Here, for $|t-x|<\delta$, we have

$$
0<\theta^{\prime} \equiv \frac{1}{2}(1+\theta(t-x) / x)^{-1 / 2} \leqq 2^{-1 / 2} .
$$

Now substituting (9) into $b_{m}(x ; t)$ and multiplying out, we obtain

$$
b_{m}(x ; t)=b_{1}{ }^{(m)}+b_{2}{ }^{(m)}+b_{3}{ }^{(m)}+O\left(m^{-2}\right),
$$

where

$$
\begin{aligned}
b_{1}^{(m)}= & 2 \cos p\left(\alpha_{m} x\right) \cos p\left(\alpha_{m} t\right) \\
b_{2}^{(m)}= & \frac{\theta^{\prime}(t-x)}{x} \cdot 2 \cos p\left(\alpha_{m} x\right) \cos p\left(\alpha_{m} t\right) \\
b_{3}^{(m)}= & \frac{\nu^{2}-\frac{1}{4}}{2}\left[1+\frac{\theta^{\prime}(t-x)}{x}\right] \\
& \cdot\left[\frac{2}{\alpha_{m}^{\prime} x} \sin p\left(\alpha_{m} x\right) \cos p\left(\alpha_{m} t\right)+\frac{2}{\alpha_{m} t} \cos p\left(\alpha_{m} x\right) \sin p\left(\alpha_{m} t\right)\right] .
\end{aligned}
$$

Denoting

$$
B_{M}(x ; t)=\sum_{m=1}^{M} b_{m}(x ; t)=\sum_{k=1}^{4} B_{k}{ }^{(M)},
$$

where $B_{k}{ }^{(M)}=\sum_{m=1}^{M} b_{k}{ }^{(m)}(k=1,2,3)$ and $B_{4}{ }^{(M)}=\sum_{m=1}^{M} O\left(m^{-2}\right)$, and together with (2) and (3), we have the difference kernel

$$
D_{M}(x ; t)=\left(B_{1}{ }^{(M)}-C_{M}\right)+B_{2}^{(M)}+B_{3}{ }^{(M)}+B_{4}{ }^{(M)} .
$$

3. Boundedness of Difference Kernel. By examining each term on the right hand side of (11), the following theorem shows that the difference kernel $D_{M}(x ; t)$ of Bessel and Fourier cosine kernels on $[0,1]$ is uniformly bounded in a neighborhood of any fixed point in $(0,1)$.

Theorem 1. $D_{M}(x ; t)$ is bounded independently $(b . i$.$) of t$ and $M$ at any fixed point $x \in(0,1)$ for $t \in N(x ; \delta)=[x-\delta, x+\delta]$, where $0<\delta \leqq$ $\min \{x / 2,(1-x) / 2\}$.

Proof. Throughout this proof, $\sum$ means $\sum_{m=1}^{M}$.

(i) $B_{4}{ }^{(M)}$ in (11) is certainly b.i. of $M$ and $t$.

(ii) To show that $B_{3}{ }^{(M)}$ is b.i. of $M$ and $t$ in $N(x ; \delta)$. Since $x \neq 0$ and $t \geqq x / 2$, it is sufficient to show that $\sum 2 \alpha_{m}{ }^{-1} \sin p\left(\alpha_{m} x\right) \cos p\left(\alpha_{m} t\right)$ and $\sum 2 \alpha_{m}{ }^{-1} \cos$ $p\left(\alpha_{m} x\right) \sin p\left(\alpha_{m} t\right)$ are b.i. of $M$ and $t$. But from (5) and trigonometric addition formulas we only have to consider

$B_{31}^{(M)} \equiv \sum \alpha_{m}{ }^{-1} \sin \left(\alpha_{m}(t+x)-a^{\prime}\right) \quad$ and $\quad B_{32}^{(M)} \equiv \sum \alpha_{m}{ }^{-1} \sin \left(\alpha_{m}(t-x)\right)$, where

$$
a^{\prime}=(2 \nu+1) \pi / 2
$$

Now by letting

$$
\eta=(t+x) / 2, \quad b=\pi(2 \nu-1)(t+x) / 4-\pi(2 \nu+1) / 2,
$$


and noting $\alpha_{m}=m \pi\left(1+O\left(m^{-1}\right)\right)$ from (6) and (7), we have

$$
\begin{aligned}
B_{31}^{(\mathcal{M})} & =\sum \alpha_{m}{ }^{-1} \sin (2 m \pi \eta+b) \cos \left(2 \eta \beta_{m}\right)+\sum \alpha_{m}{ }^{-1} \cos (2 m \pi \eta+b) \sin \left(2 \eta \beta_{m}\right) \\
& =\sum(m \pi)^{-1} \sin (2 m \pi \eta+b)+\sum O\left(m^{-2}\right) .
\end{aligned}
$$

Thus, from Lemma $1, B_{31}^{(M)}$ is b.i. of $M, \eta$ and $b$; therefore, it is b.i. of $M$ and $t$ in $N(x ; \delta)$. Next, for $t \in N(x ; \delta)$, by setting

$$
\zeta=\pi(t-x), \quad a=(2 \nu-1) / 4
$$

we similarly have

$$
\begin{aligned}
B_{32}^{(\boldsymbol{\mu})} & =\sum{\alpha_{m}}^{-1} \sin ((m+a) \zeta) \cos \left(\beta_{m} \zeta / \pi\right)+\sum{\alpha_{m}}^{-1} \cos ((m+a) \zeta) \sin \left(\beta_{m} \zeta / \pi\right) \\
& =\sum(m \pi)^{-1} \sin ((m+a) \zeta)+\sum O\left(m^{-2}\right) .
\end{aligned}
$$

It follows, from Lemma 2 , that $B_{32}^{(M)}$ is b.i. of $M, a$ and $\zeta$ for $t$ in $N(x ; \delta)$. Therefore, $B_{3}{ }^{(M)}$ is b.i. of $M$ and $t$ in $N(x ; \delta)$.

(iii) Next we consider $B_{2}{ }^{(M)}$. By a trigonometric addition formula

$$
\begin{aligned}
B_{2}{ }^{(\boldsymbol{M})} & =\left(\theta^{\prime}(t-x) / 2\right) \sum \cos \left(\alpha_{m}(t+x)-a^{\prime}\right)+\left((t-x) \theta^{\prime} / x\right) \sum \cos \left(\alpha_{m}(t-x)\right) \\
& \equiv B_{21}^{(\boldsymbol{M})}+B_{22}^{(\boldsymbol{N})} .
\end{aligned}
$$

By means of (6) and $\eta$ and $b$ defined in (13), we have $\cos \left(\alpha_{m}(t+x)-a^{\prime}\right)=\cos (2 m \pi \eta+b) \cos \left(2 \beta_{m} \eta\right)-\sin (2 m \pi \eta+b) \sin \left(2 \beta_{m} \eta\right)$. Here, noting $\sin \left(2 \beta_{m} \eta\right)=\eta\left(1-4 \nu^{2}\right)(4 m \pi)^{-1}+O\left(m^{-2}\right)$ and $\cos \left(2 \beta_{m} \eta\right)=1+$ $O\left(m^{-2}\right)$ from (7), therefore by Lemma $1, B_{21}^{(\mathcal{M})}$ is b.i. of $M$ and $t$ in $N(x ; \delta)$. Next, from (6),

$$
\begin{aligned}
B_{22}^{(\boldsymbol{\mu})}= & \left(\zeta \theta^{\prime} / \pi x\right) \sum \cos ((m+a) \zeta) \cos \left(\beta_{m} \zeta / \pi\right) \\
& -\left(\zeta \theta^{\prime} / \pi x\right) \sum \sin ((m+a) \zeta) \sin \left(\beta_{m} \zeta / \pi\right),
\end{aligned}
$$

where $a$ and $\zeta$ are defined in (14). From $\cos \left(\beta_{m} \zeta / \pi\right)=1+O\left(m^{-2}\right)$ and

$$
\left|\left(\zeta \theta^{\prime} / \pi x\right) \sum \cos ((m+a) \zeta)\right| \leqq\left|\zeta \theta^{\prime} /(\pi x \sin (\zeta / 2))\right|,
$$

which is bounded for $0<|\zeta| \leqq \pi \delta \leqq \pi / 4$, since $|(\zeta / 2) / \sin (\zeta / 2)|$ is bounded, it follows that the first sum of $B_{22}^{(M)}$ is b.i. of $M$ and $\zeta$. The second sum of $B_{22}^{(\mu)}$ is b.i. of $M$ and $\zeta$ by Lemma 2 , by noting $\sin \left(\beta_{m} \zeta / \pi\right)=\zeta\left(1-4 \nu^{2}\right)(8 m \pi)^{-1}+O\left(m^{-2}\right)$.

Hence $B_{2}{ }^{(M)}$ is b.i. of $M$ and $t$ in $N(x ; \delta)$.

(iv) Lastly, we consider $B_{1}{ }^{(M)}-C_{M}$. Write as in (ii)

$$
\begin{aligned}
B_{1}^{(M)}-C_{M}= & \sum \cos \left(\alpha_{m}(t+x)-a^{\prime}\right)+\sum \cos \left(\alpha_{m}(t-x)\right) \\
& -\left[1+\sum \cos (m \pi \pi(t+x))\right]-\sum \cos (m \pi(t-x)) \\
= & I_{1}+I_{2}-I_{3}-I_{4} .
\end{aligned}
$$

Here $I_{1}$ is bounded similarly as $B_{21}^{(\boldsymbol{N})}$ in the case (iii) and $I_{3}$ is bounded by Lemma 1 because $0<3 \delta \leqq t+x \leqq 2-3 \delta<2$; both b.i. of $M$ and $t$ in $N(x ; \delta)$. Now

$$
\begin{aligned}
I_{2}-I_{4}= & \sum \cos ((m+a) \zeta) \cos \left(\beta_{m} \zeta / \pi\right) \\
& -\sum \sin ((m+a) \zeta) \sin \left(\beta_{m} \zeta / \pi\right)-\sum \cos (m \zeta) .
\end{aligned}
$$


The boundedness of the second sum was a case in (iii). Now noting $\cos \left(\beta_{m} \zeta / \pi\right)=$ $1+O\left(m^{-2}\right)$, we can write

$$
\sum \cos ((m+a) \zeta) \cos \left(\beta_{m} \zeta / \pi\right)-\sum \cos (m \zeta)=B_{1}{ }^{*}+\sum O\left(m^{-2}\right),
$$

where

$$
\begin{aligned}
{B_{1}}^{*} & =\sum[\cos ((n \iota+a) \zeta)-\cos (m \zeta)] \\
& =-2 \sin (a \zeta / 2) \sum \sin ((m+a / 2) \zeta) .
\end{aligned}
$$

Hence, by Lemma 1,

$$
\left|B_{1}^{*}\right| \leqq|2 \sin (a \zeta / 2) / \sin (\zeta / 2)|
$$

which is bounded on $0<|\zeta| \leqq \pi \delta \leqq \pi / 4$, since $a=(2 \nu-1) / 4$. Therefore, $B_{1}{ }^{(M)}-C_{M}$ is b.i. of $M$ and $t$ in $N(x ; \delta)$.

(v) Thus, from (i)-(iv), $D_{M}(x ; t)$ is b.i. of $M$ and $t$ in $N(x ; \delta) \subset(0,1)$ with $\delta \leqq \min \{x / 2,(1-x) / 2\}$. This completes the proof of Theorem 1 .

Now we show the boundedness of $D_{\mathcal{M}}(x ; t)$ on $[0,1]$ for any fixed $x$ in $(0,1)$. From (2)

$$
C_{m} \equiv C_{M}(x ; t)=1+\sum_{m=1}^{M} \cos (m \pi(t+x))+\sum_{m=1}^{M} \cos (m \pi(t-x)),
$$

and hence by Lemma $1, C_{M}$ is b.i. of $M$ and $t$ on $[0, x-\delta] \cup[x+\delta, 1]$ for every fixed $x$ in $(0,1)$. Also from an inequality in $[7$, p. 584]

$$
\left|B_{M}(x ; t)\right| \leqq \frac{4 d^{2} t^{1 / 2}}{\pi c^{2}\left|t^{2}-x^{2}\right|(2-x-l) x^{1 / 2}},
$$

where $c$ and $d$ are positive constants, $0<x<1,0 \leqq t \leqq 1$ and $x \neq t$. Hence

$$
\left|B_{\mathbf{M}}(x ; t)\right| \leqq \frac{4 d^{2}}{\pi c^{2} \delta^{2}(1-x) x^{1 / 2}},
$$

that is, $B_{M}$ is also b.i. of $M$ and $t$ on $[0, x-\delta] \cup[x+\delta, 1]$ for fixed $x$ in $(0,1)$.

Combining the above results with Theorem 1 , we obtain

Theorem 2. $D_{M}(x ; t)$ is $b . i$. of $M$ and $t$ on $[0,1]$ for any fixed $x$ in $(0,1)$.

4. Equiconvergence. The Riemann-Lebesgue theorems for Fourier series [5, p. 403] and Bessel series [7, p. 589] are respectively as follows:

Let $f$ be Lebesgue integrable on $[0,1]$. For $x$ in $(0,1)$ and $\delta>0$, we denote $\Delta=[0,1]-(x-\delta, x+\delta)$. Then

$$
\int_{\Delta} f(t) C_{\boldsymbol{M}}(x ; t) d t \rightarrow 0 \quad \text { as } \quad M \rightarrow \infty .
$$

If $\int_{\Delta} t^{1 / 2} f(t) d t$ converges absolutely, then

$$
\int_{\Delta} f(t) B_{M}(x ; t) d t \rightarrow 0 \quad \text { as } \quad M \rightarrow \propto .
$$

Hence by taking $f(t) \equiv 1$

$$
\int_{\Delta} B_{M}(x ; t) d t \rightarrow 0 \text { as } M \rightarrow \infty .
$$


Then, by means of a general convergence theorem in [2, p. 425], (15) follows for a Lebesgue integrable function $f$.

Also from (3), we immediately obtain

$$
\int_{\Delta} f(t) D_{M}(x ; t) d t \rightarrow 0 \text { as } M \rightarrow \infty .
$$

We also need the absolute continuity of the Lebesgue integral [4, p. 148]. Let $\epsilon>0$, there exists a $\delta>0$ such that for every measurable set $e \subset E$ of measure $m e<\delta$, the inequality $\int_{e}|f(t)| d t<\epsilon$ holds.

With these preliminary results we obtain the equiconvergence theorem of Bessel and Fourier cosine series as follows.

TheOREm 3. Let $f$ be Lebesgue integrable on $[0,1]$. Then the Bessel series of $f$ is equiconvergent with its Fourier cosine series at every point $x \in(0,1)$.

Proof. Set

$$
\begin{aligned}
& B_{M}{ }^{*}(x ; f)=\int_{0}^{1} f(t) B_{M}(x ; t) d t, \\
& C_{M}{ }^{*}(x ; f)=\int_{0}^{1} f(t) C_{M}(x ; t) d t .
\end{aligned}
$$

Then we are to show that

$$
D_{M}^{*}(x ; f)=\int_{0}^{1} f(t) D_{M}(x ; t) d t \rightarrow 0 \quad \text { as } \quad M \rightarrow \infty .
$$

From Theorem 2 we know that $\left|D_{M}(x ; t)\right| \leqq K$ for all $M$ and $t \in[0,1]$ and fixed $x \in(0,1)$. For any $\epsilon>0$, from the absolute continuity of Lebesgue integral, there exists a $\delta>0$ such that

$$
\int_{\Delta^{\prime}}|f(t)| d t<\frac{\epsilon}{2 K}
$$

where $\Delta^{\prime}=[x-\delta / 2, x+\delta / 2]$. Denote $\Delta^{*}=[0,1]-\Delta^{\prime}$. Hence, by Theorem 2 and Eqs. (16) and (17),

$$
\begin{aligned}
\left|D_{M}{ }^{*}(x ; f)\right| & \leqq\left|\int_{\Delta^{\prime}} f(t) D_{M}(x ; t) d t\right|+\left|\int_{\Delta^{*}} f(t) D_{M}(x ; t) d t\right| \\
& <\epsilon / 2+\epsilon / 2=\epsilon
\end{aligned}
$$

for sufficiently large $M$, which completes the proof.

Here we notice that the cosine series may equally well be replaced by a sine or sine and cosine series.

Miami University

Oxford, Ohio

1. E. W. Hoвson, "On the representation of a function by series of Bessel functions," Proc. London Math. Soc., (2), v. 7, 1909, pp. 359-388.

2. E. W. HoBson, The Theory of Functions of a Real Variable and the Theory of Fourier Series, Vol. II, Dover, New York, 1958. MR 19, 1166.

3. K. KNopP, Theory and Application of Infinite Series, Blackie, London, 1957.

4. I. P. NATANson, Theory of Functions of a Real Variable, GITTL, Moscow, 1950; English transl., Vol. I, Ungar, New York, 1955. MR 12, 598; MR 16, 120, 804. 
5. E. C. Titchmarsh, The Theory of Functions, Oxford Univ. Press, London, 1939.

6. E. C. Titchmarsh, Eigenfunction Expansions Associated With Second-Order Differential Equations, Oxford, at the Clarendon Press, 1946; Part I, Oxford Univ. Press, London, 1962. MR 8, 458.

7. G. N. Watson, A Treatise on the Theory of Bessel Functions, 2nd ed., Cambridge Univ. Press and Macmillan, New York, 1944. MR 6, 64.

8. W. H. Young, "On series of Bessel functions," Proc. London Math. Soc., (2), v. 18, 1920, pp. 163-200.

\title{
On a Numerical Solution of an Integral Equation with Singularities
}

\author{
By Robert G. Voigt
}

1. Introduction. Annular airfoil theory gives rise to Fredholm integral equations of the second kind in the following form:

$$
f(x)=g(x)+\int_{0}^{1} G(x, y) f(y) d y, \quad 0 \leqq x \leqq 1,
$$

where the kernel $G(x, y)$ has the form

$$
G(x, y)=\int_{0}^{1} \frac{q(y, z)}{z-x} d z
$$

and $g(x)$ is a continuous function; in particular it may be of the form

$$
g(x)=\int_{0}^{1} \frac{r(x, z)}{z-x} d z .
$$

For what follows, we will assume that $q(y, z)$ and $r(x, z)$ are continuous functions as they would be in most physical problems; however, the results are valid for more general functions. By using a Fourier series technique given in Collatz [1], we are able to neatly evaluate the singular integrals involved, but as will be seen, this is not the only advantage of the technique. We also obtain a kernel function of degenerate type; that is

$$
G(x, y)=\sum_{i=1}^{n} m_{i}(x) M_{i}(y)
$$

Then the integral equation may be solved using a method applicable to degenerate kernels such as the simple one given in Mikhlin [2].

An example of the method applied to an integral equation arising in annular airfoil theory is included at the end of this paper.

2. Handling the Singularities. The first step in handling the singularities is to apply the changes of variables suggested by Collatz [1]: Let

$$
\begin{aligned}
& x=\frac{1}{2}(1+\cos \theta), \\
& y=\frac{1}{2}(1+\cos \psi), \\
& z=\frac{1}{2}(1+\cos \varphi) .
\end{aligned}
$$

Received April 22, 1965. 